\title{
DEVELOPMENT OF OPEN ABDOMEN DEVICE BASED ON BIODESIGN METHODOLOGY
}

\author{
Sierra, Manuela (1); \\ Pereira, Salín (2); \\ Isaza, Juan Felipe (1); \\ Montoya, Iván Darío (1); \\ Diaz, Christian Andrés (1); \\ Velásquez, Diego (3); \\ Londoño, Carolina (3) \\ 1: Eafit University; \\ 2: Hospital Pablo Tobón Uribe; \\ 3: CES University
}

\begin{abstract}
Open Abdomen (OA) therapy, is purposely leaving the fascial edges of the abdomen un-approximated after a laparotomy. During the OA therapy, there must be a temporary abdominal closure (TAC) device installed in the patient to achieve abdominal closure gradually without affecting its safety. However, the actual TAC devices have some gaps in terms of functionality or usability, therefore a new device is proposed. Intending to design a usable and functional technique for patients all over the world, the BioDesign Innovation Process was used. This iterative methodology focuses on healthcare needs, invention, and concept development with three main phases: Identify, Invent and Implement. At the end of these phases, the team successfully developed two new abdominal closure techniques that fill in the gaps of functionality and usability, using a simulator that realistically mimicked the physical and mechanical properties of an open abdomen. In terms of functionality, the novel techniques showed safe installation, operational security, secure grip, low invasiveness and control of intra-abdominal pressure. In terms of usability, the devices showed better results in efficiency, effectiveness and easy re-exploration than the control group.
\end{abstract}

Keywords: New product development, Design methodology, Biomedical design, 3D printing, Research methodologies and methods

\section{Contact:}

Sierra, Manuela

Eafit university

Product Design Engineering

Colombia

msierr22@eafit.edu.co

Cite this article: Sierra, M., Pereira, S., Isaza, J. F., Montoya, I. D., Diaz, C. A., Velásquez, D., Londoño, C. (2021) 


\section{INTRODUCTION}

User-centered design success cases are on the rise, and medical devices are no exception. Designing a user-centered medical device is an iterative cycle where is important to identify an unmet clinical need through understanding profoundly the users, their tasks, experience and environment. Also, define these needs and corroborate them with the user to confirm that these problems are "real." When designing a solution, the iterative process of prototyping in an agile way and continuously evaluating with the user, it is an important task to solve this need in a correct manner. However, the key to developing a successful medical device is not only to observe, design and evaluate iteratively, is also important to keep in mind at all times its implementation in the market, a strong intellectual property process and a regulatory strategy. All of this in order to design something useful for the user, striking for the market, valid for the regulatory entity and novel enough to be patented. These steps to health technology innovation have been developed, refined, and enhanced by Stanford University by the name of Biodesign Innovation Process. This process has proven its relevance across numerous clinical domains. Success cases of the implementation of this methodology are in the field of biophotonics, nephrology, radiology and audiology among others (Beswick et al., 2017) (Augustin et al., 2020) (Steinberger et al., 2017) (Sridhar et al., 2016). In this case, we will discuss how this methodology was used to develop an open abdomen therapy device.

Open Abdomen (OA) therapy, is defined as intentionally leaving the fascial edges of the abdomen unapproximated after a laparotomy surgery. Patients with an open abdomen are exposed to severe complications that lead to prolonged periods in intensive care units and often associated with significant mortality ( $27 \%$ in-hospital and $16 \%$ perioperative). During the OA therapy, there must be a temporary abdominal closure (TAC) device installed in the patient to achieve abdominal closure gradually and as soon as the patient physiologically tolerate it (See Figure 1) (Pereira-Warr \& SierraMarin, 2018). The most common causes of an OA therapy are peritonitis, trauma, vascular emergencies and hemorrhage, ischemia, pancreatitis and post-operative abdominal compartment syndrome (Coccolini et al., 2017). The high mortality rates associated with this procedure are related to its complications, such as entero-atmospheric fistula, bowel resection, contents leakage, ventral hernia and intra-abdominal abscess. This complications lead to the patient's prolonged periods of time in intensive care units, causing increased hospital costs (Cristaudo, 2017).
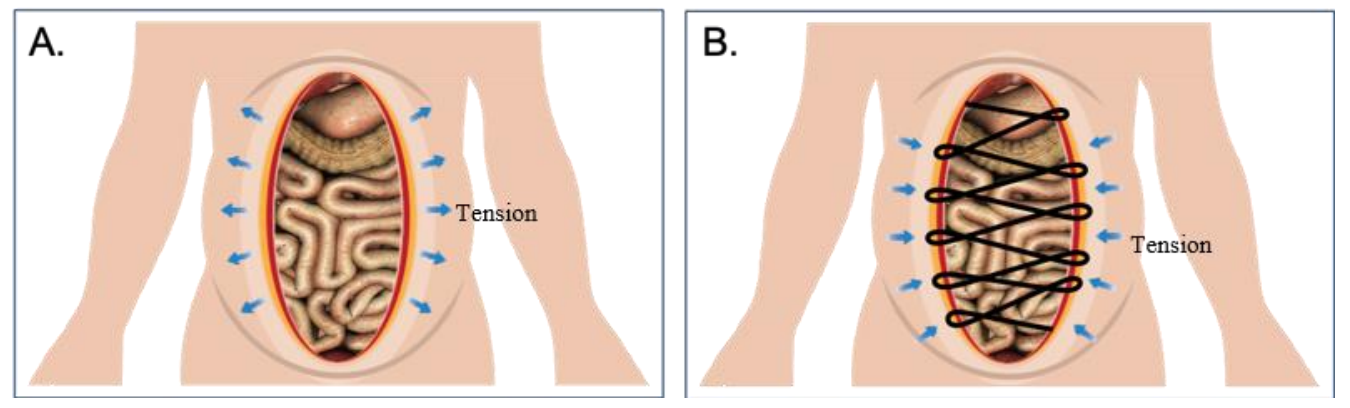

Figure 1. Open abdomen therapy $(A)$ once the abdomen is opened, the intraabdominal hypertension lowers but the muscles retract $(B)$ a TAC device must be installed to counteract the strength of the muscles and facilitate the total closure of the abdomen.

Although OA therapy evidently reduces organ failure, improving survival rate there is concern for long-term consequences. Cheatham and Safcsak (2008) demonstrated in a retrospective study, using the SF-36 health survey, that the skin-grafted abdominal viscera and incisional hernias that may result from the use of OA therapy initially reduce perceived physical, social, mental and emotional health, but after abdominal wall reconstruction this perceptions are restored to normal levels. Therefore, a TAC technique that achieves the initial closure in a gradual and healthy way, is not only important to improve physical but also social, mental and emotional health. The main characteristics of a TAC technique is to preserve the patients' safety and wellbeing at all times, prevent the development of intra-abdominal complications and achieve fascial closure at the end of OA therapy (Bjarnason, 2014). Some of the most used TAC techniques are the Bogotá Bag, the mesh, ABRA (Abdominal ReApproximation Anchor System), VAC (Vacuum-Assisted Closure) and the VAWCM (VacuumAssisted Wound Closure and Mesh). However, they still lack usability and functionality features. For 
instance, the Bogotá bag causes lacerations in the skin and can tears tissue with increased abdominal internal pressure, the same as the Mesh, which can tear the fascia causing evisceration due to its stiffness and excessive traction (Karakose et al., 2016). ABRA on the other hand, increases the risk of bowel damage while perforating the abdominal wall for the elastomer to pass and causes pressure sores in the skin which may cause long term complications due to its high invasiveness. The VAC does not allow constant visualization of the wound, might cause pain and bleeding due to continuous negative pressure, can damage the abdominal wall tissue and the frequent dressing changes may lead to mechanical injury to the bowels and cause a fistula (Bjarnason, 2014) (Kılıç et al., 2018). The combination between VAC and the mesh, VAWCM, requires frequent dressing change under general anesthesia every two or three days, or earlier to release mesh tension due to increasing IAP (Intraabdominal Pressure) caused by excessive traction. Another concern with the VAWCM technique is the traction force and trauma done to the fascial edges with the suture of the polypropylene mesh, and the risk of a tear due to its stiffness. Analyzing, these weaknesses and considering the recommendations done by the IROA (International Register of Open Abdomen), promoting future development and researches regarding less technological techniques, the need to develop a new technique emerged (Coccolini et al., 2017) (Bjarnason, 2014) (Kılıç et al., 2018). In view of these gaps, a new technique is developed considering that the criteria for choosing one technique over another should not only be based on the availability of the most advanced systems, so that a less technological technique should be easily accessed by patients around the world, thus saving more lives (Coccolini et al., 2017).

\section{MATERIALS AND METHODS}

\subsection{Designing the Temporary Abdominal Closure device}

The BioDesign Innovation Process is a robust multi-disciplinary and systematic approach based on the concept that healthcare innovation can be reproduced, taught and learned through an iterative cycle, which main focus is to observe. During the development of this methodology the user needs are always the core of concept development, with an inclination towards creating and testing so that the team can keep learning and improving upon the initial ideas. Also, it highlights the regulatory dimension that needs to be considered in the context of implementation into a healthcare system. This process evaluates healthcare needs, aims for invention and concept development, it can be applied to any field of medicine including OA therapy. The process incorporates three main phases (i) Identify (ii) Invent (iii) Implement, within which there are other stages. In this particular case, the development of the TAC device is in the first stage of the implementation phase (G. Yock et al., 2015) (See Figure 2).

During the Identify phase, researchers discover problems and opportunities, read related bibliography and get immersed in the clinical environment to recognize the most crucial needs, using techniques such as observation, interviews, and surveys to the physicians, nurses, staff, patients, and families. In this case, the observations were realized at the Hospital Pablo Tobón Uribe and CES clinic in Medellín, Colombia. During these visits, there was special attention for inefficiencies, malfunctions, and risks in the process, also during the post-operative care where stress, pain, or uncertainty occur, which helped understand even more about the real opportunities for improvement. During the multiple visits, some issues were identified: (i) During the installation of the devices surgeons must be very careful because they can easily pierce the intestine, (ii) the grip was sometimes unstable and could be unclasp with sudden increases in pressure. (iii) When surgeons needed to tighten or loosen the device, it was a complex and painful process that often involved anesthesia and an operating room. (iv)When a wound control was necessary, the device had to be completely uninstalled which also involved anesthesia and an operating room. (v) The uninstallation of most of the devices was painful to watch even for observers, there was a lot of bleeding due to adhesion of the devices to the intestines and the abdominal wall. Therefore, the need statement that identifies what is required to address the problems is: to develop a dynamic abdominal closure device that is usable for the surgeon and has a functional design. After the need is identified, the second half of this phase is to validate it and determine which specifications will help to solve it. With this, researchers begin to cooperate with experts, in an iterative cycle of validation and observation. Subsequently, the identified specifications were evaluated in terms of the clinical context, market characteristics, stakeholders, and current technologies. Then, the specifications were characterized in a Product Design Specification (PDS) 
(See Table 1), which was essential for the design and engineering team to keep in mind at all times the goal and scope of the project (Wall, Wynne, \& Krummel, 2015).

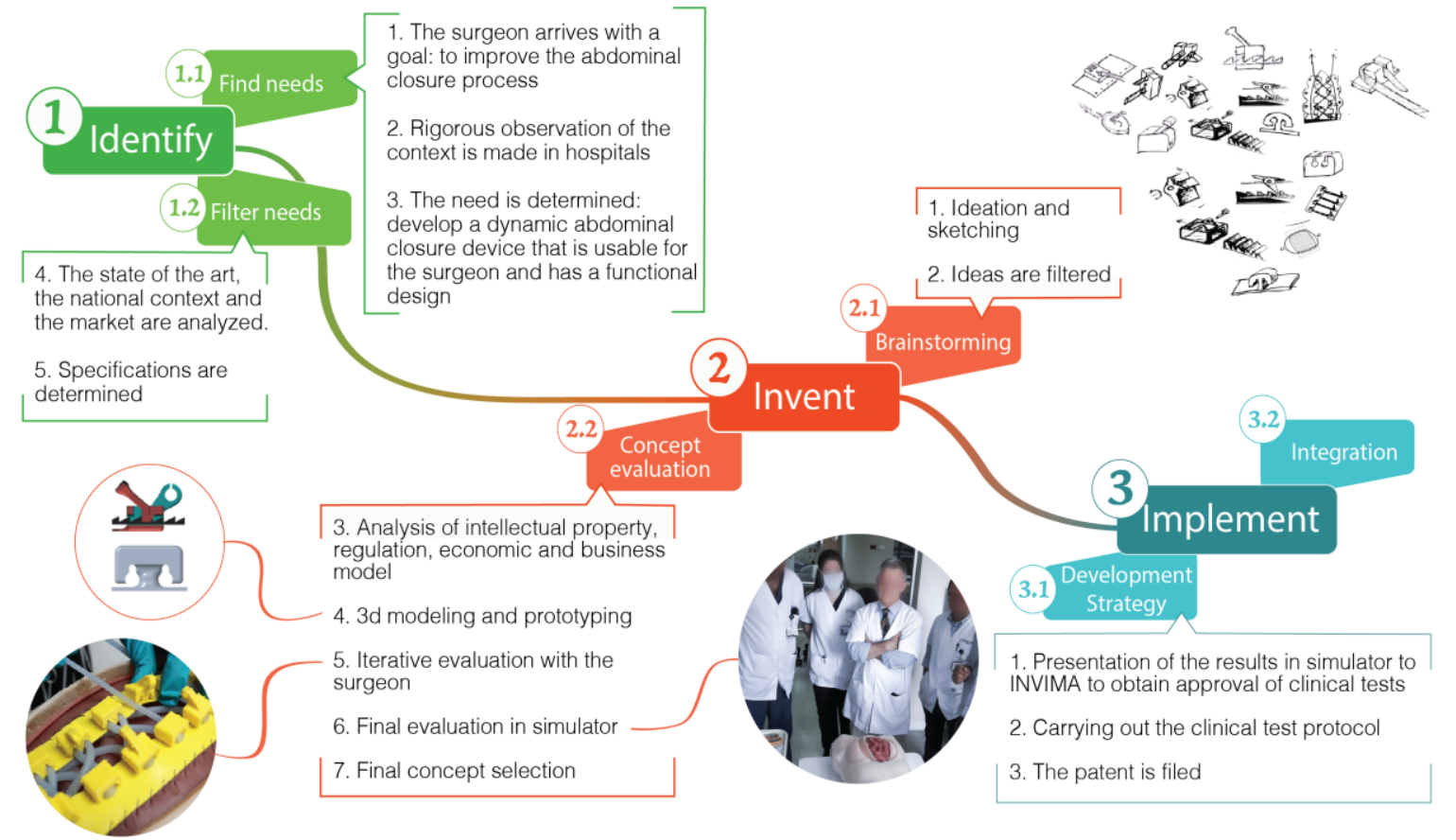

Figure 2. BioDesign innovation process applied to the novel TAC device

Table 1. Product design specifications for new TAC device

\begin{tabular}{|c|c|c|c|c|c|c|}
\hline Aspect & Attribute & Priority & Criteria & $\begin{array}{l}\text { Ideal } \\
\text { value }\end{array}$ & Metrics & Validation tool \\
\hline \multirow{5}{*}{ 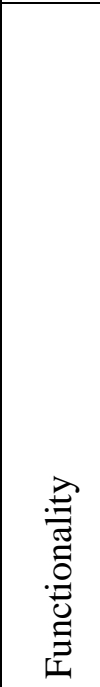 } & Safe installation & High & $\begin{array}{c}\text { Number of perforations done } \\
\text { to the polyvinyl with the } \\
\text { needle }\end{array}$ & $<1$ & \# & $\begin{array}{r}\text { Simulator/ } \\
\text { Observation }\end{array}$ \\
\hline & $\begin{array}{l}\text { Operational } \\
\text { security }\end{array}$ & High & $\begin{array}{c}\text { Number of perforations done } \\
\text { to the polyvinyl with the } \\
\text { device }\end{array}$ & 0 & $\#$ & $\begin{array}{r}\text { Simulator/ } \\
\text { Observation }\end{array}$ \\
\hline & Secure grip & High & $\begin{array}{l}\text { Number of tears during } \\
\text { traction }\end{array}$ & 0 & $\#$ & $\begin{array}{r}\text { Simulator/ } \\
\text { Observation }\end{array}$ \\
\hline & $\begin{array}{l}\text { Low } \\
\text { invasiveness }\end{array}$ & Medium & $\begin{array}{l}\text { Number of abdominal wall } \\
\text { layers that get in perforated }\end{array}$ & $0-1$ & \# & $\begin{array}{r}\text { Simulator/ } \\
\text { Observation }\end{array}$ \\
\hline & $\begin{array}{l}\text { Control of intra- } \\
\text { abdominal } \\
\text { pressure }\end{array}$ & Medium & $\begin{array}{l}\text { The pressure difference } \\
\text { before and after traction }\end{array}$ & $2<x<6.5$ & Hgmm & $\begin{array}{r}\text { Simulator/ } \\
\text { Mobile device }\end{array}$ \\
\hline \multirow{6}{*}{ 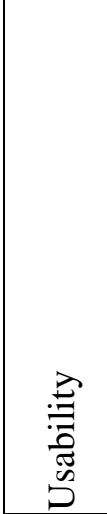 } & \multirow{2}{*}{ Efficiency } & High & Installation time & $<600$ & $\mathrm{Sec}$ & Simulator/Timer \\
\hline & & High & Traction time & $<240$ & $\mathrm{Sec}$ & Simulator/Timer \\
\hline & \multirow{3}{*}{ Effectiveness } & High & Ease of installation & $\geq 3.5$ & $\begin{array}{l}\text { Scale } 1 \\
\text { to } 5\end{array}$ & Simulator/Survey \\
\hline & & High & Ease of closure & $\geq 3.5$ & $\begin{array}{l}\text { Scale } 1 \\
\text { to } 5\end{array}$ & Simulator/Survey \\
\hline & & High & Ease of opening & $\geq 3.5$ & $\begin{array}{l}\text { Scale } 1 \\
\text { to } 5\end{array}$ & Simulator/Survey \\
\hline & \begin{tabular}{|l|} 
Easy \\
re-exploration
\end{tabular} & Medium & Wound visibility at all times & YES & Yes/No & $\begin{array}{r}\text { Simulator/ } \\
\text { Observation }\end{array}$ \\
\hline
\end{tabular}


The team chose eight specifications that support the general objective, to develop a dynamic abdominal closure device that is usable for the surgeon and has a functional design. These specifications were evaluated using a physical simulator of an open abdomen. In this manuscript usability means the way that the device will be used and whether it enables the surgeon to do the job in a satisfying, simple and effective manner. Stating so, three specifications were determined to help the device achieve usability: (i) efficiency: is referred to as quick installation and traction times and is considered to be achieved when the device is installed in less than 10 minutes (the average time of what a device like the Mesh takes) and achieves traction in less than 2 minutes (the average time of what a device like ABRA takes). (ii) Effectiveness: is the ease of installing, opening and closing the device and is measured in a 1 to 5 scale depending on the ease of installation, opening and closure, stating that a score of 3.5 or higher is acceptable. (iii) Ease of re-exploration: is when the wound is visible at all times so that the surgeon can take a quick look now and then to monitor the state of the wound, this specification can be easily answered with a yes or no. On the other hand, functionality is defined as the quality of the device to be helpful, practical and right for closing an open abdomen. To accomplish functionality requirements the device must be (iv) safe to install: when the polyvinyl is not perforated with the needle while suturing. (v) Have operational security: safe to operate when after installed the device by itself does not perforate the polyvinyl. (vi) Have secure grip: when no component of the simulator tears down during traction. (vii) Have low invasiveness: the capacity of the device to be installed in just one abdominal wall layer without complications. (viii) Control of the intra-abdominal pressure: when the pressure difference among the initial intra-abdominal pressure and the one after traction is significant $(>1 \mathrm{mmHg})$, however, it does not exceed $10 \mathrm{mmHg}$, where significant alterations in organ function can be occur (Hunter \& Damani, 2004).

During the Invent phase, the needs are used as a guidance but there is no limit for creativity and the team brainstormed in various sessions. In this case, there were around 50 to 60 sketched concepts that went through a filtering stage, grouped into similar categories, to allow similar and overlapping ideas to either be linked or eliminated. This also helped to identify areas that needed to be explored deeper. There were a few subsequent brainstorming sessions to strengthen the concepts to meet the criteria, while also providing interesting and potentially novel solutions. Also, the analysis of the intellectual property, regulation, economic and possible business model was carried out. Next, the concepts were 3D modeled and 30 different designs were taken to the prototyping stage and tested with a surgeon in an iterative cycle of creation and testing. During this phase, the goal of testing was to fine-tune details through feedback but without metrics being measured. This early-stage testing and research, made the team choose two designs which were the most promising ones, denominated DACT (Dynamic Abdominal Closure Technique) and PACT (Pawl Abdominal Closure Technique). Both were taken to the last evaluation phase, which consisted on testing the devices in the simulator with 25 experienced surgeons before the clinical trial on human beings, using The Mesh as a control group (See Figure 3). The previously validated simulator's main purpose was to recreate the lifelike conditions of an OA. The goal of the evaluation was to determine which was the most viable device in terms of functionality and usability to take to de pre-clinical trial. Each trial was made individually on the same simulator, all the participants used the same mesh brand (Winer Surgical Mesh), surgical tools (scissors, clamps and forceps), suture (Non-absorbable monofilament \#0) and the same polyvinyl type to protect the synthetic bowels just like in real life. A device was chosen randomly, and the intra-abdominal pressure of the simulator was set to a correlated intra-abdominal hypertension of Grade III (21-25 mmHg). During the study, the variables recorded were: (i) installation time, (ii) traction time, (iii) number of perforations done to the polyvinyl with the needle, (iv) number of perforations done to the polyvinyl with the device, (v) number of tears during traction and (vi) the visibility of the wound was made by observation by a team member. Pressure before traction and pressure after traction were tracked with a mobile device connected to the simulator using Bluetooth communication. Finally, a survey was made to address concerns regarding ease of installation, opening and closure of the new devices to measure its effectiveness, on a scale from 1 to 5,1 being the lowest and 5 the highest score. 

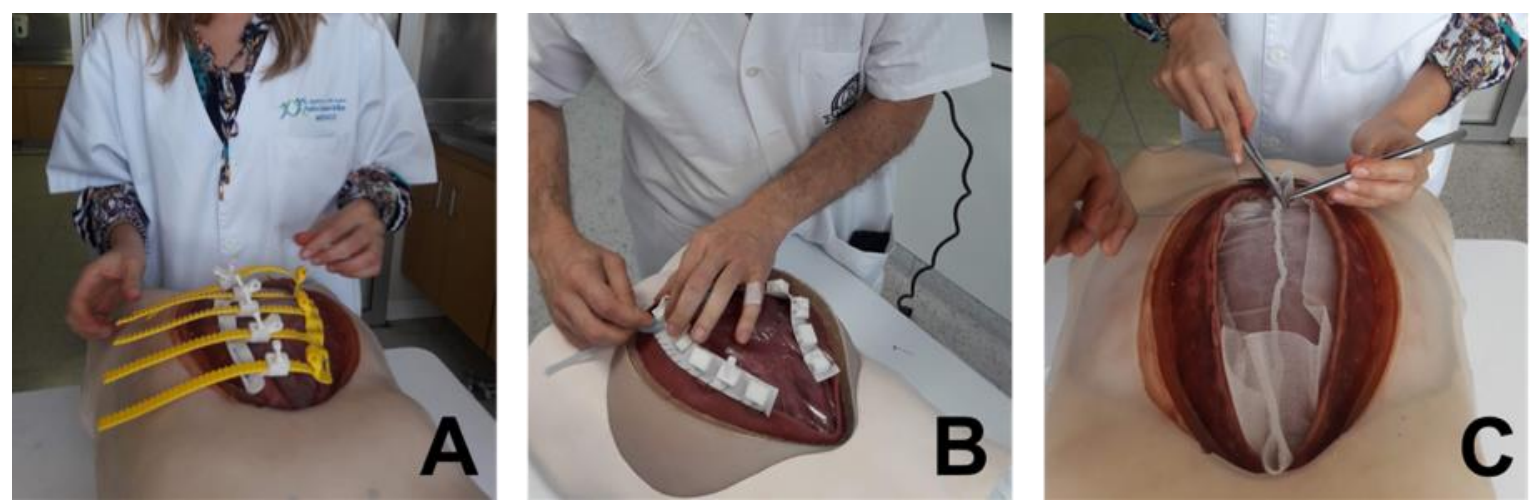

Figure 3. Evaluation of (A) PACT, (B) DACT and (C) the mesh on the physical simulator by surgeons

The results on the simulator were useful for the implementation phase, were a regulatory strategy and a detailed plan for device testing was developed including pre-clinical and clinical trials. An In-depth analysis of each concept was undertaken to choose one to proceed after both concepts underwent laborious background research. This includes a comprehensive analysis of the evaluation on the simulator and understanding the engineering feasibility, resources, and personnel needed to undergo the industrial scaling (Schwartz et al., 2016)(Wall et al., 2015).

The pre-clinical trial would be performed with a total of 40 patients, 20 for the experimental and 20 for the control group (VAC). Then, an external committee will evaluate the frequency of successful abdominal closure to approve the clinical trial with 84 patients (42 experimental -42 control). The clinical trial is a prospective and controlled open multicenter study to compare the number of days of successful abdomen closure when using the VAC and when using DACT in adult patients who undergo laparotomy at the Hospital Pablo Tobón Uribe and CES clinic in Medellín, Colombia. Also, to identify the complications presented during the use of both devices for abdominal closure and to determine the satisfaction of surgeons with the use of the devices. For this, three formats were developed to record the study variables, one format when entering, another during the patient's stay and another at the end of the OA therapy.

Also, after identifying which aspect made this TAC device novel and useful, researching the field and making sure the was no invention that overlapped with it and choosing the type of protection, the patent for the device was filed.

\section{RESULTS}

Both devices interfere with only one abdominal wall layer, the fascia, making them minimally invasive. Also, they allow easy re-exploration, with the wound visible at all times, with simple mechanisms that make it easy to install and uninstall. Therefore, the devices can be adapted to changing intra-abdominal pressures without taking the patient to the operating room. DACT has two flexible 3D printed bands that are sutured to both edges of the fascia, each band has grips every centimeter and there are silicone medical hoses that go around a grip at each side and keep the closure dynamic. PACT, on the other side, is a fully 3D printed device that consists of two components: a flexible band that goes sutured to the one fascial edge and has strips distributed every centimeter, with pawl-like texture and a flexible band that is sutured to the other fascial edge and has releasable heads, where the strips are inserted and removed easily with consistent performance.

The evaluation of the functionality and usability of the three devices was carried out with ANOVA tests using Brown-Forsythe and Welch corrections for unequal variances. Also, Dunett T3 post-hoc test was used for multiple comparisons to determine statistically different groups. Prism 8 (version 8.4.3 (471)) was used for the statistical analysis. Level of significance *p-value $<0.05$.

From the twenty-five surgeons in various stages of their career that participated in the study, the average years of expertise performing OA surgeries was 9 and $76 \%$ reported to have performed more than $50 \mathrm{OA}$ surgeries. Results of functionality and usability are summarized in Table 2, and showed that DACT and PACT devices satisfied all the ideal value ranges for the evaluated criteria. In terms of functionality, the simulator's abdominal wall with lifelike hardness and bowels helped verify that the devices didn't have elements that could harm any kind of tissue. Also, both devices could be sutured to the fascia without 
complications, reducing its invasiveness and during traction, the novel devices did not induce tears of the fascia. Both novel devices allowed the control of the intra-abdominal pressure, however, none of the values for the novel devices were significantly different from those found with the implanted surgical mesh. On the other hand, statistical analysis in terms of usability showed that although the installation time did not differ among the devices, the ANOVA test for traction time was different between the evaluated devices. Additionally, post-hoc analysis showed that the traction times for each of the novel devices was significantly lower than the traction time for the surgical mesh (pDACT $<1.0 \mathrm{E}-4$ and pPACT $<1.0 \mathrm{E}-4$ ), positively impacting the efficiency of installation. All the other scores showed that DACT and PACT devices were easy to install, open, and close. Finally, both of the novel devices allowed for a constant visibility of the wound, a feature that cannot be obtained with the use of the surgical mesh.

Table 2. Statistical analysis

\begin{tabular}{|c|c|c|c|c|c|c|c|c|c|}
\hline 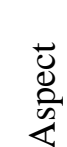 & $\begin{array}{l}\text { Design } \\
\text { specifications }\end{array}$ & Ideal & $\begin{array}{l}\text { DACT } \\
\text { average }\end{array}$ & $\begin{array}{l}\text { PACT } \\
\text { average }\end{array}$ & $\begin{array}{c}\text { Mesh } \\
\text { average }\end{array}$ & $\begin{array}{l}\text { ANOV } \\
\text { A test }\end{array}$ & p-value & $\begin{array}{l}\text { Significa } \\
\text { nce }\end{array}$ & $\begin{array}{r}\text { Adjusted } \\
\text { p-value }\end{array}$ \\
\hline \multirow[b]{5}{*}{ 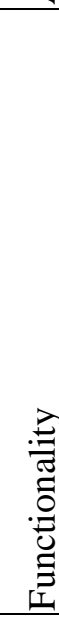 } & \begin{tabular}{|l} 
Safe \\
installation
\end{tabular} & $\leq 1$ & 0.2 & 0.2 & 0.4 & $\begin{array}{c}\mathrm{F}: \\
0.8208 \\
\end{array}$ & 0.4447 & NS & \\
\hline & $\begin{array}{l}\text { Bowel } \\
\text { protection }\end{array}$ & 0 & 0.0 & 0.0 & 0.0 & - & - & NS & \\
\hline & Secure grip & 0 & 0.0 & 0.0 & 0.0 & $\begin{array}{c}\mathrm{F}: \\
1.000\end{array}$ & 0.3837 & NS & \\
\hline & $\begin{array}{l}\text { Perforation of } \\
\text { the abdominal } \\
\text { wall }\end{array}$ & $0-1$ & 1.0 & 1.0 & 1.0 & - & - & NS & \\
\hline & $\begin{array}{l}\text { Control of } \\
\text { intra- } \\
\text { abdominal } \\
\text { pressure } \\
\end{array}$ & $\begin{array}{c}2<x< \\
6.5\end{array}$ & 2.9 & 3.2 & 2.1 & $\begin{array}{c}\mathrm{F}: \\
2.030\end{array}$ & 0.139 & NS & \\
\hline \multirow{7}{*}{ 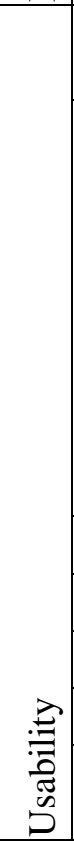 } & $\begin{array}{l}\text { Efficiency- } \\
\text { Installation } \\
\end{array}$ & $<600$ & 522.3 & 587.1 & 590.6 & $\begin{array}{c}F: \\
0.9877 \\
\end{array}$ & 0.3799 & NS & \\
\hline & $\begin{array}{l}\text { Efficiency- } \\
\text { Traction }\end{array}$ & $<240$ & 107.9 & 41.8 & 235.8 & $\begin{array}{c}\mathrm{F}: \\
74.72\end{array}$ & 0.0001 & $\begin{array}{l}\text { Significa } \\
\text { ntly } \\
\text { different }\end{array}$ & $\begin{array}{r}\text { DACT } \\
\text { vs. PACT } \\
(<0.0001) \\
\text { DACT } \\
\text { vs. Mesh } \\
(<0.0001) \\
\text { PACT vs. } \\
\text { Mesh } \\
(<0.0001) \\
\end{array}$ \\
\hline & \multicolumn{9}{|l|}{ Effectiveness } \\
\hline & Ease to install & $>3.5$ & 4.0 & 4.3 & - & & & & \\
\hline & Ease to open & $>3.5$ & 4.3 & 4.3 & - & & & & \\
\hline & Ease to close & $>3.5$ & 4.5 & 4.4 & - & & & & \\
\hline & $\begin{array}{l}\text { Easy re- } \\
\text { exploration }\end{array}$ & YES & Yes & Yes & No & - & - & & \\
\hline
\end{tabular}

\section{DISCUSSION}

Open abdomen is a worldwide used therapy to treat multiple pathologies that otherwise would be mortal. Current techniques for OA procedures vary in their technology, therefore in their usability and functionality. The Bogotá bag causes damage in the skin and doesn't have a secure grip. The surgical Mesh, can occasionally tear from the fascia due to excessive traction or its stiffness when the patient 
coughs, vomits, or sneezes (Karakose et al., 2016). ABRA causes pressure sores in the skin, increasing its invasiveness. The VAC causes pain and bleeding due to continuous negative pressure (Bjarnason, 2014) (Kılıç et al., 2018). And the combination between VAC and the mesh, VAWCM, requires frequent dressing change under general anesthesia increasing its cost. Analyzing these weaknesses, the present study shows the development of two novel devices designed using the BioDesign methodology (Coccolini et al., 2017) (Bjarnason, 2014) (Kılıç et al., 2018).

The novel devices designed and evaluated in the present study, DACT and PACT, were intended to overcome most of the current problems associated with TAC technologies. Characteristics such as efficiency, effectiveness, intra-abdominal pressure control, easy re-exploration, low invasiveness, secure grip, safe installation and operational security were constantly perfected through an iterative design and evaluation phase on the OA simulator. This simulator realistically mimicked the physical and mechanical properties of an open abdomen. Being able to take both designs so far, was possible due to this tool, otherwise the iterative testing and redesigning would have led to a long and expensive process in animals or cadavers. In the simulator, the devices were compared with a surgical mesh with 25 experienced surgeons. Results of the study suggested that both devices are suitable for applications in OA procedures, as they were comparable or had advantages over the surgical mesh in terms of usability and functionality. After the quantitative analysis of the results in a simulator, a qualitative analysis was also made with the comments in the survey. With both analysis, it was determined that DACT would be the design chosen for the pre-clinical and clinical trials. Due to its versatility, ease of sterilization, geometry that is easy to produce on an industrial scale and the use of commercial parts (the silicone hose) that will probably reduce its price. In addition, it is a device that would perform well in adverse events, due to the flexible silicones which were a secure option for sudden IAP increases and having them as a separate part, which can easily be replaced without being traumatic. On the other hand, if a single PACT tie bursts, it would be necessary to change half of the device, leading to a more traumatic and expensive situation. However, achieving fascial closure at the end of OA therapy, without complications and in a few days are really important functionality aspects that can only be proven in-vivo, for instance, future clinical studies are warranted.

\subsection{Implications in the design practice}

Being able to develop a dynamic abdominal closure device that is usable for the surgeon and has a functional design was possible with the BioDesign Innovation Process. This methodology is a clear guidance to solve healthcare needs, with a step-by-step process that lets the team evaluate decisions and prototypes constantly. Developing an open abdomen device without the user being the center of the design would have probably led to failure. What made this project successful was observing from the perspective of the patient, the surgeons and the nurses. Asking the most basic questions at every stage to understand what they say, do, see, touch and feel was a key element to this development.

From the present work, is once again validated how well designed and thought this methodology is. Even without prior knowledge of medicine, a team made up mostly of designers and engineers was able to design a medical device that is currently in the process of being patented and validated by INVIMA (the homologous of the FDA in Colombia). All this with the continuous accompaniment of a surgeon (a potential user), with whom the fluid and constant communication was an important aspect to the evaluation of the concepts and prototypes. By allowing early corrections, the development becomes more promising, making clinical trials less complex and less expensive, impacting directly on the innovation in healthcare devices. Moreover, students, designers and engineers are encouraged to look at a broad range of areas, keeping in mind that deep expertise in a field is not a requirement. Often people who are deeply immersed in the area fail to see the opportunities and needs that surround them, since they are used to do things a certain way, see the same scenario on a daily basis and repeat experiences. However, being rigorous observers, with capabilities in design and engineering, unexplored areas can be easily identified.

\section{REFERENCES}

Augustin, D.A., Chertow, G.M. and Azagury, D.E. (2020), "Innovation in hemodialysis: Using the Biodesign process to identify unmet needs", Journal of Vascular Access, pp. 1-6.

Beswick, D.M., Kaushik, A. and Beinart, D. (2017), "Biomedical device innovation methodology: applications 
Bjarnason, T. (2014), Open Abdomen Therapy with Vacuum-Assisted Wound Closure and Mesh-Mediated Fascial Traction, available at: http://lup.lub.lu.se/record/4221708.

Cheatham, M.L. and Safcsak, K. (2008), "Longterm Impact of Abdominal Decompression: A Prospective Comparative Analysis", available at:https://doi.org/10.1016/j.jamcollsurg.2008.05.008.

Coccolini, F., Montori, G., Ceresoli, M., Catena, F., Ivatury, R., Sugrue, M., Sartelli, M., et al. (2017), "IROA: International Register of Open Abdomen, preliminary results", World Journal of Emergency Surgery, BioMed Central, Vol. 12 No. 1, p. 10.

Cristaudo, A. (2017), "Complications and Mortality Associated with Temporary Abdominal Closure Techniques : A Systematic Review and Meta-Analysis", No. May.

Karakose, O., Fatih Benzin, M., Pülat, H., Zafer Sabuncuoglu, M., Eken, H., Zihni, I. and Barut, I. (2016), "Bogota Bag Use in Planned Re-Laparotomies.", Medical Science Monitor : International Medical Journal of Experimental and Clinical Research, International Scientific Information, Inc., Vol. 22, pp. 2900-4.

Kılıç, E., Uğur, M., Yetim, İ. and Temiz, M. (2018), "Effects of temporary abdominal closure methods on mortality and morbidity in patients with open abdomen”, Ulusal Travma ve Acil Cerrahi Dergisi, Vol. 24 No. 4, pp. 321-326.

Schwartz, J.G., Kumar, U.N., Azagury, D.E., Brinton, T.J. and Yock, P.G. (2016), "Needs-Based Innovation in Cardiovascular Medicine: The Stanford Biodesign Process", JACC: Basic to Translational Science, Elsevier, Vol. 1 No. 6, pp. 541-547.

Sridhar, S., Madhuri K R, Shah, S., Chaturvedi, J., Vijayarajan, A. and Appaji, A.M. (2016), “A biodesign based study on chronic tinnitus and evaluation of adaptive noise cancellation technology in its management", 2016 8th International Conference on Communication Systems and Networks (COMSNETS), IEEE, pp. 1-6.

Steinberger, J.D., Denend, L., Azagury, D.E., Brinton, T.J., Makower, J. and Yock, P.G. (2017), "Needs-Based Innovation in Interventional Radiology: The Biodesign Process", Techniques in Vascular and Interventional Radiology, Vol. 20 No. 2, pp. 84-89.

Wall, J., Wynne, E. and Krummel, T. (2015), "Biodesign process and culture to enable pediatric medical technology innovation”, Seminars in Pediatric Surgery, Elsevier, Vol. 24 No. 3, pp. 102-106. 


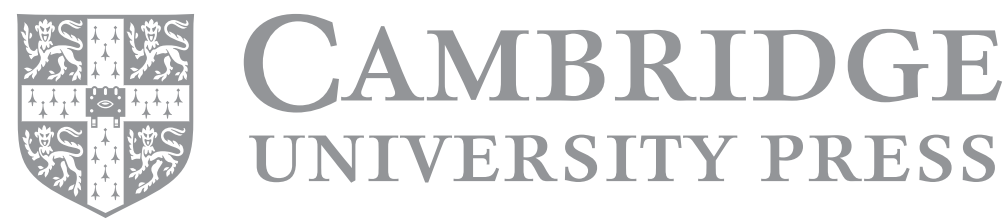

